\title{
Radiation use efficiency, N accumulation and biomass production of high-yielding rice in aerobic culture
}

\section{$\operatorname{AUTHOR}(S):$}

Katsura, Keisuke; Okami, Midori; Mizunuma, Hiroaki; Kato, Yoichiro

\section{CITATION:}

Katsura, Keisuke ...[et al]. Radiation use efficiency, $\mathrm{N}$ accumulation and biomass

production of high-yielding rice in aerobic culture. Field Crops Research 2010, 117(1): 81 89

\section{ISSUE DATE:}

2010-05-08

URL:

http://hdl.handle.net/2433/109967

\section{RIGHT:}

(c) 2010 Elsevier B.V.; この論文は出版社版でありません。引用の際には 出版社版をご確認ご利用ください。; This is not the published version. Please cite only the published version. 
Table 1. $\mathrm{N}$ accumulation rate $\left(\mathrm{kgN} \mathrm{ha}^{-1} \mathrm{~d}^{-1}\right)$ during the reproductive stage (from 9-week after sowing to heading) and ripening stage for each cultivar.

\begin{tabular}{|c|c|c|c|c|c|c|c|c|}
\hline & \multicolumn{4}{|c|}{ Reproductive stage } & \multicolumn{4}{|c|}{ Ripening stage } \\
\hline & \multicolumn{2}{|c|}{ Tokyo } & \multicolumn{2}{|c|}{ Osaka } & \multicolumn{2}{|c|}{ Tokyo } & \multicolumn{2}{|c|}{ Osaka } \\
\hline & 2007 & 2008 & 2007 & 2008 & 2007 & 2008 & 2007 & 2008 \\
\hline \multicolumn{9}{|l|}{ Aerobic } \\
\hline Akihikari & 2.56 & 3.19 & 4.08 & 2.71 & 0.78 & 0.79 & 1.46 & 2.29 \\
\hline IRAT109 & 2.58 & 3.49 & 3.44 & 3.27 & 0.63 & 0.86 & 1.33 & 1.20 \\
\hline Lemont & 2.38 & 2.26 & 3.07 & 2.89 & 0.97 & 1.44 & 1.09 & 0.91 \\
\hline Takanari & 2.69 & 4.04 & 2.88 & 3.23 & 0.71 & 1.25 & 1.19 & 0.37 \\
\hline Average & 2.55 & 3.25 & 3.36 & 3.02 & 0.77 & 1.08 & 1.27 & 1.19 \\
\hline \multicolumn{9}{|l|}{ Flooded } \\
\hline Akihikari & 2.99 & 2.58 & 2.53 & 3.82 & 0.02 & 1.02 & 0.93 & 1.20 \\
\hline IRAT109 & 2.71 & 2.90 & 1.32 & 2.20 & 0.28 & 0.20 & 1.33 & 1.48 \\
\hline Lemont & 2.22 & 1.89 & 1.55 & 1.77 & 0.77 & 0.59 & 0.60 & 1.12 \\
\hline Takanari & 3.50 & 2.54 & 2.66 & 2.40 & 1.26 & 2.00 & 0.71 & 1.38 \\
\hline Average & 2.86 & 2.48 & 2.01 & 2.55 & 0.58 & 0.95 & 0.89 & 1.30 \\
\hline \multicolumn{9}{|l|}{ LSD (0.05) } \\
\hline Water regime & 0.27 & 0.31 & 0.50 & 0.34 & NS & NS & 0.24 & NS \\
\hline Cultivar & 0.38 & 0.44 & 0.71 & 0.48 & NS & 0.29 & 0.34 & 0.30 \\
\hline Cultivar $\times$ Water & NS & NS & NS & 0.68 & NS & 0.41 & NS & 0.42 \\
\hline
\end{tabular}


Table 2. Aboveground biomass at maturity, growth duration, incident and intercepted radiation, fraction of radiation intercepted, and radiation use efficiency at Tokyo in 2007 and 2008.

\begin{tabular}{|c|c|c|c|c|c|c|}
\hline & $\begin{array}{c}\text { Aboveground } \\
\text { biomass at } \\
\text { maturity } \\
\left(\mathrm{t} \mathrm{ha}^{-1}\right)\end{array}$ & $\begin{array}{c}\text { Growth } \\
\text { duration }^{\mathrm{a}} \\
\text { (days) }\end{array}$ & $\begin{array}{l}\text { Incident } \\
\text { radiation }^{\mathrm{b}} \\
\left(\times 10^{3} \mathrm{MJ}\right)\end{array}$ & $\begin{array}{l}\text { Intercepted } \\
\text { radiation }^{\mathrm{b}} \\
\left(\times 10^{3} \mathrm{MJ}\right)\end{array}$ & $\begin{array}{l}\text { Fraction of } \\
\text { radiation } \\
\text { intercepted }^{\mathrm{b}}\end{array}$ & $\begin{array}{l}\text { Radiation } \\
\text { use } \\
\text { efficiency }^{\mathrm{b}} \\
\left.\text { (g MJ }^{-1}\right)\end{array}$ \\
\hline \multicolumn{7}{|l|}{2007} \\
\hline \multicolumn{7}{|l|}{ Aerobic } \\
\hline Akihikari & 13.2 & 137 & 1.67 & 1.02 & 0.61 & 1.33 \\
\hline IRAT109 & 15.0 & 147 & 1.76 & 1.19 & 0.68 & 1.34 \\
\hline Lemont & 15.6 & 161 & 1.90 & 1.28 & 0.67 & 1.24 \\
\hline Takanari & 16.1 & 171 & 2.00 & 1.30 & 0.65 & 1.30 \\
\hline Average & 15.0 & 154 & 1.83 & 1.20 & 0.653 & 1.30 \\
\hline \multicolumn{7}{|l|}{ Flooded } \\
\hline Akihikari & 13.7 & 137 & 1.67 & 1.03 & 0.61 & 1.39 \\
\hline IRAT109 & 13.3 & 147 & 1.76 & 1.11 & 0.63 & 1.29 \\
\hline Lemont & 13.1 & 161 & 1.90 & 1.23 & 0.65 & 1.13 \\
\hline Takanari & 23.5 & 168 & 1.99 & 1.37 & 0.69 & 1.68 \\
\hline Average & 15.9 & 153 & 1.83 & 1.19 & 0.647 & 1.37 \\
\hline \multicolumn{7}{|l|}{ LSD (0.05) } \\
\hline Water regime & 0.4 & & & 0.01 & 0.006 & 0.03 \\
\hline Cultivar & 0.6 & & & 0.02 & 0.01 & 0.04 \\
\hline Cultivar $\times$ Water & 0.8 & & & 0.02 & 0.01 & 0.06 \\
\hline \multicolumn{7}{|l|}{2008} \\
\hline \multicolumn{7}{|l|}{ Aerobic } \\
\hline Akihikari & 15.1 & 145 & 1.72 & 1.08 & 0.63 & 1.43 \\
\hline IRAT109 & 17.8 & 145 & 1.72 & 1.20 & 0.70 & 1.50 \\
\hline Lemont & 17.4 & 159 & 1.87 & 1.29 & 0.69 & 1.35 \\
\hline Takanari & 23.6 & 169 & 1.98 & 1.37 & 0.69 & 1.72 \\
\hline Average & 18.5 & 155 & 1.82 & 1.23 & 0.68 & 1.50 \\
\hline \multicolumn{7}{|l|}{ Flooded } \\
\hline Akihikari & 15.1 & 138 & 1.64 & 1.06 & 0.65 & 1.47 \\
\hline IRAT109 & 13.3 & 142 & 1.70 & 1.12 & 0.66 & 1.32 \\
\hline Lemont & 14.0 & 157 & 1.84 & 1.20 & 0.65 & 1.21 \\
\hline Takanari & 20.7 & 162 & 1.90 & 1.34 & 0.70 & 1.50 \\
\hline Average & 15.8 & 150 & 1.77 & 1.18 & 0.67 & 1.37 \\
\hline \multicolumn{7}{|l|}{ LSD (0.05) } \\
\hline Water regime & 0.5 & & & 0.01 & 0.01 & 0.05 \\
\hline Cultivar & 0.7 & & & 0.02 & 0.01 & 0.07 \\
\hline Cultivar $\times$ Water & 1.0 & & & 0.02 & 0.01 & 0.10 \\
\hline
\end{tabular}

a Values from sowing to maturity.

b Values from transplanting to maturity 
Table 3. Aboveground biomass at maturity, growth duration, incident and intercepted radiation, fraction of radiation intercepted, and radiation use efficiency at Osaka in 2007 and 2008.

\begin{tabular}{|c|c|c|c|c|c|c|}
\hline & $\begin{array}{c}\text { Aboveground } \\
\text { biomass at } \\
\text { maturity } \\
\left(\mathrm{t} \mathrm{ha}^{-1}\right) \\
\end{array}$ & $\begin{array}{l}\text { Growth } \\
\text { duration }^{\mathrm{a}} \\
\text { (days) }\end{array}$ & $\begin{array}{l}\text { Incident } \\
\text { radiation }^{\mathrm{b}} \\
\left(\times 10^{3} \mathrm{MJ}\right)\end{array}$ & $\begin{array}{l}\text { Intercepted } \\
\text { radiation }^{\mathrm{b}} \\
\left(\times 10^{3} \mathrm{MJ}\right)\end{array}$ & $\begin{array}{l}\text { Fraction of } \\
\text { radiation } \\
\text { intercepted }^{\mathrm{b}}\end{array}$ & $\begin{array}{l}\text { Radiation } \\
\text { use } \\
\text { efficiency }^{\mathrm{b}} \\
{\left(\mathrm{g} \mathrm{MJ}^{-1}\right)}\end{array}$ \\
\hline \multicolumn{7}{|l|}{2007} \\
\hline \multicolumn{7}{|l|}{ Aerobic } \\
\hline Akihikari & 15.3 & 124 & 1.80 & 1.16 & 0.64 & 1.42 \\
\hline IRAT109 & 17.5 & 134 & 1.98 & 1.54 & 0.78 & 1.24 \\
\hline Lemont & 17.5 & 148 & 2.17 & 1.70 & 0.78 & 1.13 \\
\hline Takanari & 21.7 & 155 & 2.28 & 1.69 & 0.74 & 1.28 \\
\hline Average & 18.0 & 140 & 2.06 & 1.52 & 0.737 & 1.27 \\
\hline \multicolumn{7}{|l|}{ Flooded } \\
\hline Akihikari & 14.2 & 116 & 1.68 & 1.09 & 0.65 & 1.40 \\
\hline IRAT109 & 13.1 & 126 & 1.85 & 1.21 & 0.65 & 1.20 \\
\hline Lemont & 12.6 & 137 & 2.06 & 1.38 & 0.67 & 1.06 \\
\hline Takanari & 18.9 & 142 & 2.13 & 1.54 & 0.72 & 1.31 \\
\hline Average & 14.7 & 130 & 1.93 & 1.31 & 0.674 & 1.24 \\
\hline \multicolumn{7}{|l|}{ LSD (0.05) } \\
\hline Water regime & 0.7 & & & 0.04 & 0.023 & NS \\
\hline Cultivar & 1.0 & & & 0.06 & 0.03 & 0.06 \\
\hline Cultivar $\times$ Water & 1.5 & & & 0.09 & 0.05 & NS \\
\hline \multicolumn{7}{|l|}{2008} \\
\hline \multicolumn{7}{|l|}{ Aerobic } \\
\hline Akihikari & 15.2 & 132 & 1.99 & 1.13 & 0.57 & 1.34 \\
\hline IRAT109 & 16.7 & 135 & 2.03 & 1.26 & 0.62 & 1.38 \\
\hline Lemont & 16.1 & 142 & 2.12 & 1.36 & 0.64 & 1.28 \\
\hline Takanari & 20.8 & 166 & 2.43 & 1.68 & 0.69 & 1.27 \\
\hline Average & 17.2 & 144 & 2.14 & 1.36 & 0.63 & 1.32 \\
\hline \multicolumn{7}{|l|}{ Flooded } \\
\hline Akihikari & 14.0 & 120 & 1.78 & 1.21 & 0.68 & 1.26 \\
\hline IRAT109 & 13.9 & 126 & 1.88 & 1.28 & 0.68 & 1.18 \\
\hline Lemont & 13.5 & 138 & 2.08 & 1.44 & 0.69 & 1.05 \\
\hline Takanari & 19.5 & 137 & 2.08 & 1.52 & 0.73 & 1.33 \\
\hline Average & 15.2 & 130 & 1.95 & 1.36 & 0.70 & 1.20 \\
\hline \multicolumn{7}{|l|}{ LSD (0.05) } \\
\hline Water regime & 0.7 & & & NS & 0.03 & 0.07 \\
\hline Cultivar & 1.0 & & & 0.08 & 0.04 & 0.09 \\
\hline Cultivar $\times$ Water & NS & & & 0.12 & NS & 0.13 \\
\hline
\end{tabular}

a Values from sowing to maturity.

b Values from transplanting to maturity 
Table 4. The parameters of $a$ and $b$ for the Eq. (1) (critical N\% $=a T D W^{-b}$ ) for rice grown in aerobic and flooded culture.

\begin{tabular}{|c|c|c|c|}
\hline & Logarithm of $a$ & $a$ & $\boldsymbol{b}$ \\
\hline \multicolumn{4}{|l|}{ Tokyo } \\
\hline Aerobic & $1.24 \pm 0.114$ & 3.47 & $0.36 \pm 0.050$ \\
\hline Flooded & $1.09 \pm 0.129$ & 2.98 & $0.34 \pm 0.058$ \\
\hline \multicolumn{4}{|l|}{ Osaka } \\
\hline Aerobic & $1.03 \pm 0.104$ & 2.80 & $0.28 \pm 0.046$ \\
\hline Flooded & $0.99 \pm 0.116$ & 2.69 & $0.36 \pm 0.054$ \\
\hline She ehy et al. (1998) & 1.64 & 5.18 & 0.52 \\
\hline
\end{tabular}

Values followed by \pm represent $95 \%$ confidence limits. 